\title{
Synthetic MRI of Preterm Infants at Term-Equivalent Age: Evaluation of Diagnostic Image Quality and Automated Brain Volume Segmentation
}

(D)T. Vanderhasselt, (D). Naeyaert, (D) N. Watté, (D).-J. Allemeersch, (DS. Raeymaeckers, (D). Dudink, (D). de Mey, and

(D) H. Raeymaekers

\begin{abstract}
BACKGROUND AND PURPOSE: Neonatal MR imaging brain volume measurements can be used as biomarkers for long-term neurodevelopmental outcome, but quantitative volumetric MR imaging data are not usually available during routine radiologic evaluation. In the current study, the feasibility of automated quantitative brain volumetry and image reconstruction via synthetic MR imaging in very preterm infants was investigated.
\end{abstract}

MATERIALS AND METHODS: Conventional and synthetic TIWIs and T2Wls from 111 very preterm infants were acquired at termequivalent age. Overall image quality and artifacts of the conventional and synthetic images were rated on a 4-point scale. Legibility of anatomic structures and lesion conspicuity were assessed on a binary scale. Synthetic MR volumetry was compared with that generated via MANTiS, which is a neonatal tissue segmentation toolbox based on T2WI.

RESULTS: Image quality was good or excellent for most conventional and synthetic images. The 2 methods did not differ significantly regarding image quality or diagnostic performance for focal and cystic WM lesions. Dice similarity coefficients had excellent overlap for intracranial volume (97.3\%) and brain parenchymal volume (94.3\%), and moderate overlap for CSF (75.6\%). Bland-Altman plots demonstrated a small systematic bias in all cases $(1.7 \%-5.9 \%)$

CONCLUSIONS: Synthetic TIWI and T2WI sequences may complement or replace conventional images in neonatal imaging, and robust synthetic volumetric results are accessible from a clinical workstation in less than 1 minute. Via the above-described methods, volume assessments could be routinely used in daily clinical practice.

ABBREVIATIONS: BPV = brain parenchymal volume; SyMRI = synthetic MR imaging

D espite improvements in neonatal care, a high number of infants born very preterm develop sensorimotor, cognitive, and behavioral disabilities later in life. ${ }^{1}$ Identifying children at risk is essential for adequate parental counseling and may also facilitate early intervention strategies. ${ }^{2}$ Numerous studies have shown that structural MR imaging results at term-equivalent age are predictive of future motor and cognitive outcomes, ${ }^{3-5}$ and multiple volumetric MR imaging studies have identified associations between reduced

Received December 23, 2019; accepted after revision March 16, 2020. From the Department of Radiology (T.V., M.N., N.W., G.-J.A., S.R., J.d.M., H.R.), Vrije Universiteit Brussels, Universitair Ziekenhuis Brussels, Brussels, Belgium; Department of Neonatology (J.D.), Wilhelmina Children's Hospital/Utrecht University Medical Center, Utrecht, the Netherlands; and Rudolf Magnus Brain Center (.D.), Utrecht University Medical Center, Utrecht, the Netherlands.

Please address correspondence to Tim Vanderhasselt, MD, Universitair Ziekenhuis Brussels, Department of Radiology, Laarbeeklaan 101, 1090 Brussels, Belgium; e-mail: tim.vanderhasselt@uzbrussel.be

Indicates article with supplemental on-line tables.

http://dx.doi.org/10.3174/ajnr.A6533 brain volume and impaired neurodevelopment. ${ }^{6-8}$ Notably however, quantifying brain volumes in neonates is challenging.

A neonate's head is only a quarter of the size of an adult's brain, and the relative signal intensity of the GM and WM is reversed due to the high amount of unmyelinated WM. ${ }^{9}$ Neonatal scans often demonstrate changes in the brain, such as moderate-to-severe dilation or distortion of the ventricles, hyperintensity or hypointensity zones, and cystic or hemorrhagic lesions; therefore, they require substantial adjustments to the segmentation approaches used in adults. ${ }^{10}$ Several studies have demonstrated the feasibility of neonatal MR volumetry with custom-made software. ${ }^{11}$ These methods are often time-consuming and require specialized infrastructure, technical expertise, and powerful computing resources however and are thus not available in most centers. ${ }^{9}$ These difficulties hinder the use of this important biomarker in clinical practice.

Synthetic MR imaging (SyMRI; SyntheticMR, Linköping, Sweden) is an emerging imaging technique that simultaneously quantifies R1 and R2 relaxation rates, proton density, and $\mathrm{B}_{1}$ field based on a fast, multidelay, multiecho acquisition. ${ }^{12}$ Dedicated synthetic MR imaging (SyMRI) software (Synthetic MR, Linköping, Sweden) then facilitates the reconstruction of synthetic images with 
a combination of virtually any TE, TR, and TI. This may save time because multiple synthetic sequences can be reconstructed from the same multidelay, multiecho sequence even after the patient has left the scanner. SyMRI also provides fully automated volumetric analysis based on the expected quantitative values for different brain tissue types. ${ }^{13}$ The software is integrated into the radiology PACS system, and brain volume analysis is available in $<1$ minute. ${ }^{14}$

In 1 large, prospective, multicenter, multireader trial, the overall diagnostic quality of synthetic images in adults was reportedly similar to that of conventional imaging. ${ }^{15}$ Furthermore, synthetic MR imaging segmentation is reportedly rapid and robust and exhibits excellent repeatability. ${ }^{13,14,16-18}$ To date, only a few studies have examined the feasibility of synthetic MR imaging in children however, and there are very few data available on neonates. ${ }^{17-22}$ Notably, McAllister et $\mathrm{al}^{18}$ and Kim et $\mathrm{al}^{17}$ reported that in neonates, large parts of the brain parenchyma were incorrectly labeled as CSF by SyMRI.

In the current study, we used a prototype version of SyMRI (Version 11.1; https://www.syntheticmr.com/) that was adapted to perform fast and accurate volumetry in neonates. A neonate's brain is far more hydrated than an adult brain, which caused earlier versions of SyMRI to detect a CSF haze throughout the entire brain. In the new Version 11.1, the CSF contribution in the immature brain is suppressed. Because the WM definition remains at adult values, the erroneous CSF is converted nearly entirely to GM. Therefore, in the present study, total brain volume and intracranial volume were evaluated, but further distinction between GM and WM was not attempted.

The primary objective of the current study was to evaluate the image quality and diagnostic performance of synthetic MR imaging in a neonatal population compared with conventionally acquired images. A secondary objective was to assess the new and improved SyMRI algorithm for neonatal brain segmentation and its applicability in clinical practice. To validate these segmentations, the synthetic tissue volumes were compared with data derived from MANTiS (Morphologically Adaptive Neonatal Tissue Segmentation), (http:// developmentalimagingmcri.github.io/mantis) a state-of-the-art research method for neonatal brain segmentation. ${ }^{10}$

\section{MATERIALS AND METHODS}

\section{Study Population}

A total of 111 very preterm infants (born at $<32$ weeks' gestation) were scanned at an average postmenstrual age of $40.0 \pm 1.0$ weeks at our institution (Universitair Ziekenhuis Brussels) as part of their routine clinical follow-up between February 2017 and June 2019. All patients who had both synthetic multidelay, multiecho, and conventional 2D or 3D MR imaging sequences were included in the study. Both 2D and 3D T2WI datasets were available for 59 neonates. Seventeen neonates had only the 2D T2WI dataset, while 32 neonates had only the 3D T2WI dataset.

Image quality and diagnostic performance were assessed on 76 subjects (mean gestational age at birth, $29.2 \pm 2.6$ weeks; 42 boys and 34 girls) who had both synthetic multidelay, multiecho, and conventional 3D T1WI and 2D T2WI TSE data available for comparison. Because evaluation of image artifacts and image quality was part of the experimental design, no subjects were excluded on this basis.
A conventional 3D T2WI dataset was required for segmentation using MANTiS. Ninety-one neonates (mean gestational age at birth, $29.4 \pm 2.0$ weeks; 49 boys and 42 girls) underwent both 3D T2WI TSE and multidelay, multiecho scanning and were included in the quantitative volumetric validation.

After visual quality control, 16 subjects were rejected due to the following: excessive movement during the multidelay, multiecho scan $(n=5)$, failed watershed skull-stripping by MANTiS $(n=4)$, segmentation error in MANTiS caused by a previously unreported bug $(n=6)$, or failed synthetic segmentation due to corrupted DICOM data $(n=1)$. The 75 remaining subjects included 40 boys and 35 girls with a mean gestational age at birth of $29.0 \pm 2$ weeks and a mean postmenstrual age at scanning of $40 \pm 1$ week.

\section{MR Imaging Acquisition}

All examinations were performed on a 3T Ingenia MR imaging scanner (Philips Healthcare, Best, the Netherlands) with a 16channel head coil. The children were sedated using oral chloral hydrate $(25-50 \mathrm{mg} / \mathrm{kg}$ ) and placed in a MedVac vacuum immobilization device (CFI Medical Solutions, Fenton, Michigan) before scanning. Neonatal earmuffs (Minimuffs; Natus Medical, San Carlos, California) covered by headphones were used for ear protection. Informed parental consent was obtained before MR imaging and sedation.

First, conventional images were obtained in accordance with the standard dedicated neonatal MR imaging protocol used at our institution. This included, among other things, a 3D MPRAGE and a T2WI sequence (transversal T2WI TSE, 3D T2WI, or both when time allowed). A multidelay, multiecho acquisition covering the entire brain was then acquired to perform quantitative mapping of T1, T2, and proton density using synthetic MRI. Detailed imaging parameters are shown in On-line Table 1.

Synthetic T1WIs and T2WIs and their inversion recovery variants (T1 phase-sensitive inversion recovery and T2 STIR) were generated on the basis of R1, R2, and proton density maps by fitting the data to the analytic curves describing the signal intensity, using a least-squares approach. This method has been described in detail elsewhere. ${ }^{12} \mathrm{TE}$, TR, and TI were chosen to maximize tissue contrast (On-line Table 2).

\section{Image Quality and Diagnostic Performance}

The image quality and diagnostic performance of conventional and synthetic images were assessed by a blinded senior pediatric neuroradiologist (T.V.), a neuroradiologist (S.R.), and a fellow in neuroradiology (G.-J.A.) with 12, 7, and 3 years of experience in neuroradiology, respectively. For each rater, the order of the datasets was randomized, and datasets were divided into 2 sessions with a mix of conventional and synthetic datasets in each session. Only the synthetic or the conventional images of a subject were presented in the same session. Both sessions were conducted at least 2 weeks apart to prevent recall bias.

The overall image quality for each series was assessed on a 4point Likert scale, in which poor indicates severe image-quality issues precluding diagnosis; sufficient, moderate image-quality issues but acceptable for diagnosis; good, only minor image-quality issues; and excellent, no noticeable image-quality issues. Artifacts were scored on a similar 4-point scale (severe, moderate, minor, and 
none). Lesion conspicuity and the legibility of different anatomic structures were evaluated for each conventional and synthetic dataset on a binary scale, as indicated in Table 1. Assessment of diagnostic performance included the detection of focal WM lesions and cystic degeneration. Conventional and synthetic datasets were compared with the original radiology report on the basis of all available MR images and knowledge of the clinical findings.

\section{MR Volumetry Calculations}

Synthetic tissue volumes were calculated from R1, R2, and proton density maps using the automated segmentation tool in SyMRI,

Table 1: Image quality

\begin{tabular}{lc}
\hline \multicolumn{1}{c}{ Category } & Assessment Scale \\
\hline Overall image quality & $\begin{array}{c}\text { Poor, sufficient, good, } \\
\text { excellent } \\
\text { Severe, moderate, minor, } \\
\text { none }\end{array}$ \\
Virtifacts & Legible, illegible \\
Visualization of anatomic & \\
structures & \\
Posterior cross-roads & \\
(T2/T2 STIR only) & \\
Central sulcus & \\
Lentiform nucleus & \\
Ventrolateral thalamus & \\
Dorsal pons & \\
Diagnostic performance & Present, absent \\
Focal WM lesions & \\
Cystic degeneration & \\
\hline
\end{tabular}

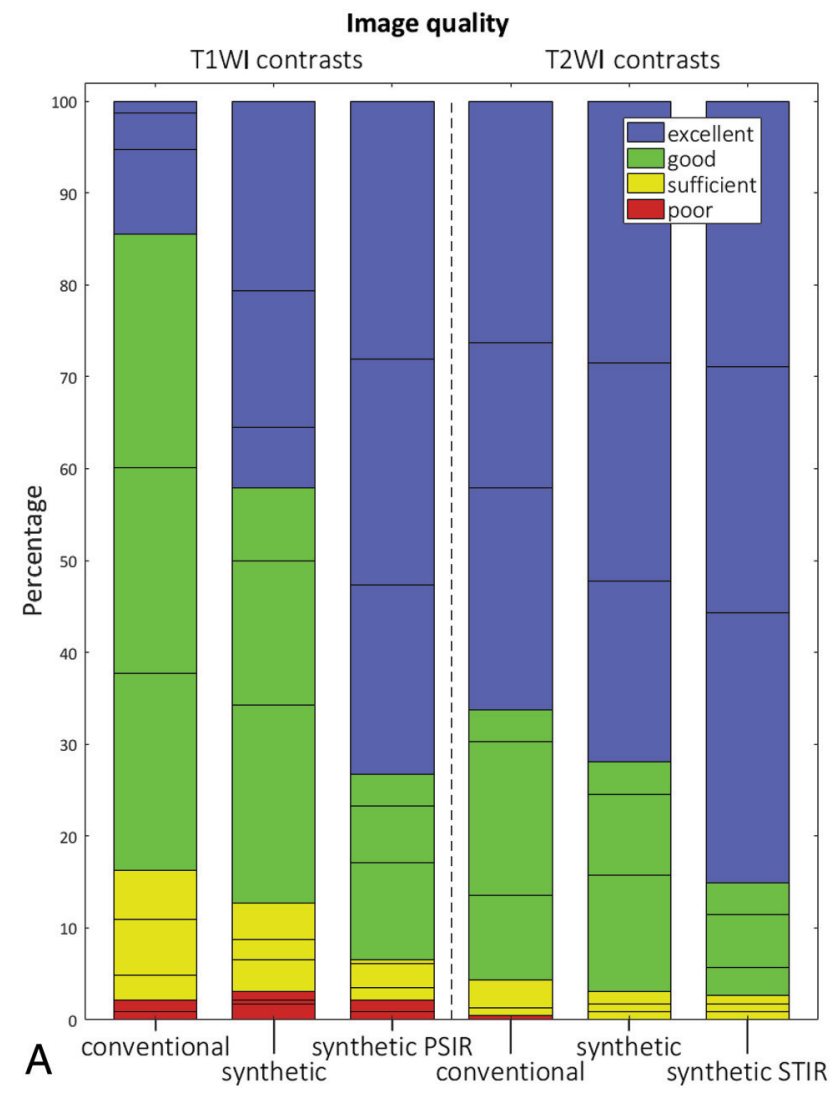

Version 11.1, optimized for segmenting neonatal brains. Segmentation was performed by comparing R1, R2, and proton density values of individual voxels with a lookup table. ${ }^{12}$ This process enables rapid segmentation, capable of coping with partial volume effects and anatomic distortions such as hydrocephalus or cysts. Synthetic MR imaging brain segmentation was validated by comparing the results with those of MANTiS, a state-of-the-art toolbox based on SPM12 (http://www.fil.ion.ucl. ac.uk/spm/software/spm12) and ITK-SNAP 1.6 (www.itksnap. org) dedicated to segmentation of the neonatal brain. ${ }^{10}$ Brain extraction was performed before tissue segmentation using the watershed-based method provided in MANTiS.

\section{Statistics}

Only good or excellent images are desirable in daily clinical practice; therefore, we chose to dichotomize image quality and artifact scores into poor-sufficient and good-excellent groups. Group differences for conventional and synthetic imaging sets were assessed with the McNemar test for all binary results, using SPSS Statistics 23 (IBM, Armonk, New York). Descriptive statistics were used to assess the legibility of anatomic structures.

Bland-Altman plots for brain parenchymal volume (BPV), brain parenchymal fraction, intracranial volume, and CSF were generated using Matlab 2016b (MathWorks, Natick, Massachusetts) to compare the measured volumes and identify systematic differences between the proposed synthetic method and MANTiS. The synthetic T2WIs were coregistered to

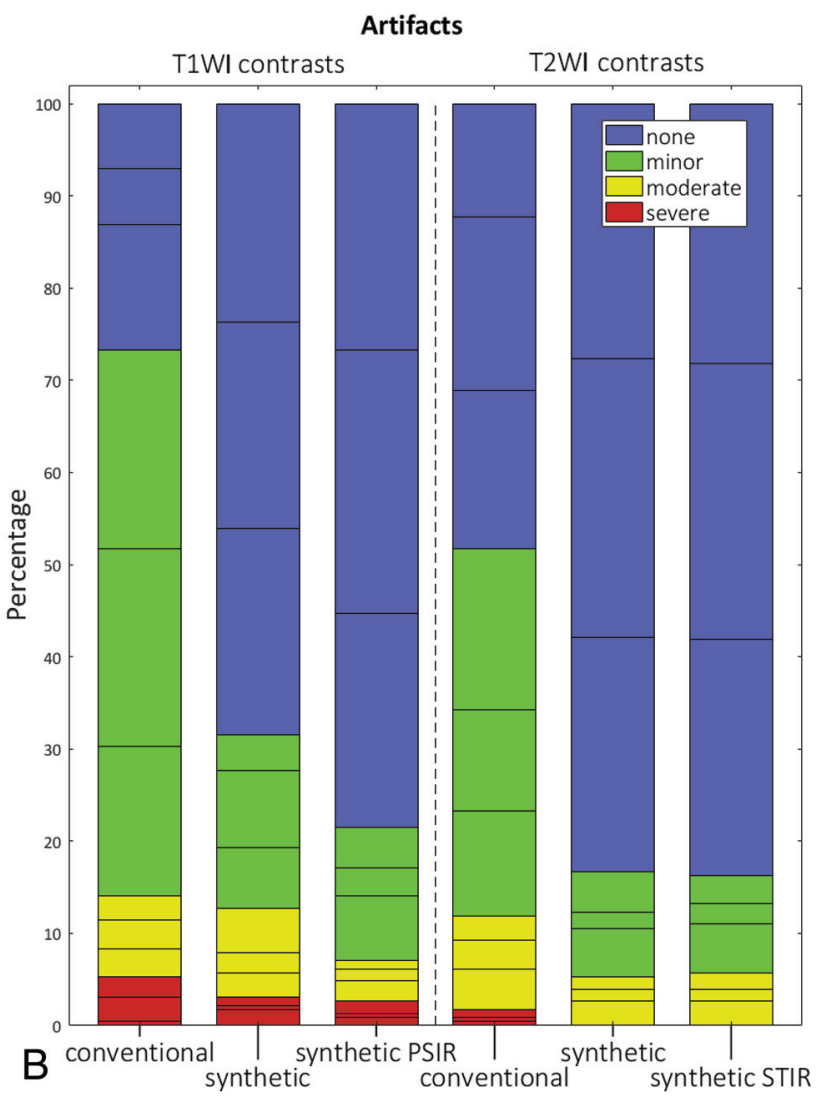

FIG 1. Qualitative comparison of TTWI and T2WI quality $(A)$ and image artifacts (B). Horizontal lines within colored portions indicate scoring per rater. PSIR indicates phase-sensitive inversion recovery. 


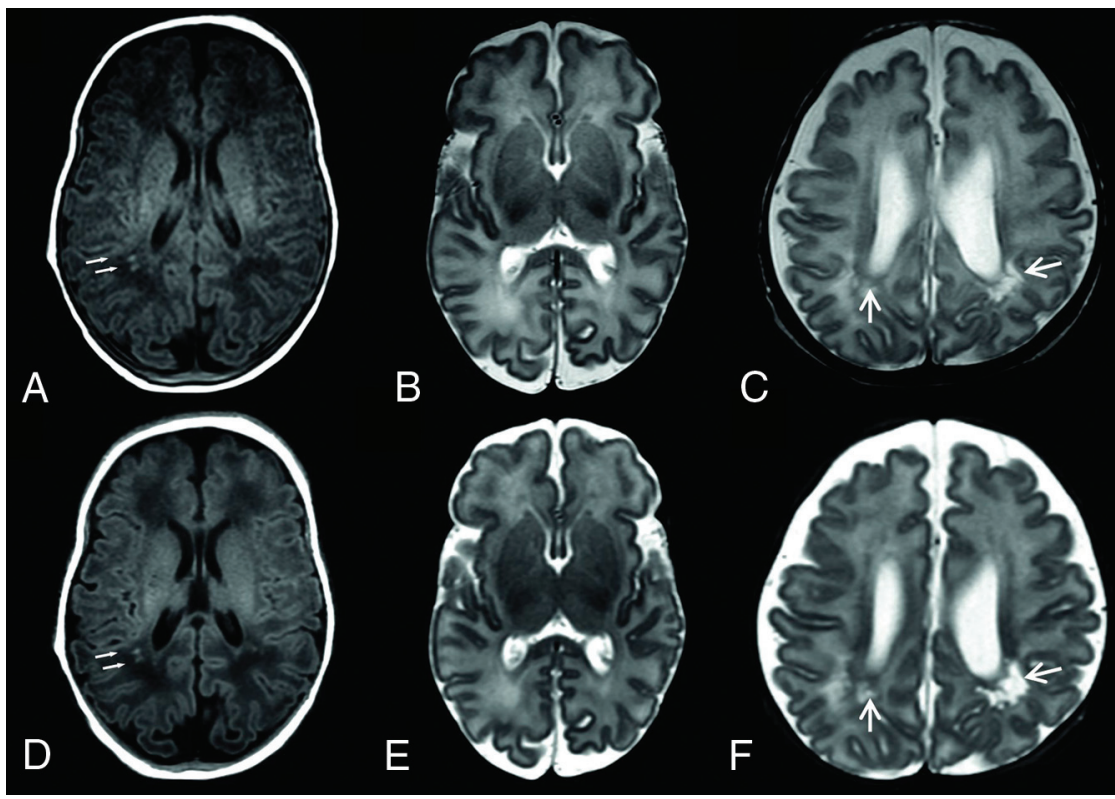

FIG 2. Upper row, Conventional TIWI ( $A$ ) and T2WI ( $B$ and $C$ ). Lower row, Synthetic TIWI (D) and T2WIs ( $E$ and $F$ ). Focal WM lesions (small arrows) are clearly visible on both TIWIs. Cystic periventricular leukomalacia (arrows) is seen around the ventricles in both T2Wls.

Table 2: Lesion conspicuity

\begin{tabular}{lcccc}
\hline & Sensitivity & Specificity & Accuracy & $P$ Value \\
\hline WM lesions $(n=23 / 76)$ & & & & \\
$\quad$ Conventional & $91 \%$ & $86 \%$ & $89 \%$ & 1.00 \\
Synthetic & $89 \%$ & $77 \%$ & $86 \%$ & \\
Cysts $(n=7 / 76)$ & & & & \\
Conventional & $96 \%$ & $57 \%$ & $92 \%$ & .29 \\
Synthetic & $100 \%$ & $64 \%$ & $95 \%$ & \\
\hline
\end{tabular}

regarding artifacts. The synthetic $\mathrm{T} 1$ phase-sensitive inversion recovery, synthetic T2W1, and synthetic T2 STIR produced significantly more images with minimal or minor artifacts $(P=.017, \quad P=.008$, and $P=.016$, respectively) than the conventional images. The most marked difference, however, was the higher percentage of synthetic images with no or minimal artifacts $(P<.001$ for all comparisons).

\section{Diagnostic Performance}

A representative sample of conventional and synthetic images is shown in Fig 2. The anatomic structures outlined in Table 1 were identified in all the conventional and synthetic sequences. The accuracy of the detection of WM lesions was slightly better in the conventional image sets, whereas the detection of cysts was better in the synthetic images; but neither of these differences was statistically significant (Table 2).

\section{Volumetric Validation}

The results of the intracranial volume, $\mathrm{BPV}$, parenchymal fraction, and CSF volume measurements are shown in the Bland-Altman plots in Fig 3. There was no correlation in the Bland-Altman plots between the mean metric under consideration and the relative difference. There was a small systematic bias the conventional dataset using SPM12 running on Matlab 2016b. The coregistration parameters were applied to the synthetic segmentation maps. A Dice similarity coefficient (DSC in the formula below) was then calculated in Matlab 2016b for CSF, BPV, and intracranial volume to investigate differences between the segmentation methods. ${ }^{23}$ This score represents the overlap between 2 segments. For the component $x, D S C_{x}$ is defined as

$$
D C_{x}=2 \mid \frac{\text { Synt }_{x} \cap 3 D_{x} \mid}{\left|S y n t_{x}\right|+\left|3 D_{x}\right| .}
$$

\section{RESULTS}

\section{Image Quality and Artifacts}

The results of the image-quality evaluation are presented in Fig 1. The number of scans rated as poor was negligible for both the conventional images and the synthetic images $(0.0 \%-2.2 \%)$. The synthetic $\mathrm{T} 1$ phase-sensitive inversion recovery resulted in significantly more images of good or excellent quality $(93.4 \%)$ than the conventional T1 $(83.8 \%)(P=.002)$, but no other statistically significant differences were detected between synthetic and conventional images when they were dichotomized into a good or excellent group and a poor or sufficient group. A similar pattern was evident in all cases. SyMRI estimated the CSF volume to be $4.50 \%$ lower than in MANTiS, while intracranial volume, BPV, and parenchymal fraction were slightly larger $(1.68 \%$ for parenchymal fraction, $4.19 \%$ for intracranial volume, and $5.87 \%$ for the BPV). Therefore, it is essential to consider which method is used for volumetry when interpreting the results. Except for the CSF measurement, the spread between both measures was small $(<3.5 \%)$, indicating good agreement between the methods. For CSF, the spread was more substantial at $18 \%$. This difference may be attributable to the smaller volume of CSF, for which minor differences in the segmentation threshold may have a greater impact. However, the spread of the relative CSF fraction (2.8\%) was much lower.

Regarding overlap of segmentation maps, the average Dice similarity coefficients were $0.9731 \pm 0.0004$ for intracranial volume, $0.9428 \pm 0.0007$ for BPV, and $0.7566 \pm 0.0055$ for CSF. The very high scores for intracranial volume and BPV indicate substantial overlap. For the CSF maps, the overlap was moderate. Figure 4 shows a representative example of the overlap of 2 CSF segments.

\section{DISCUSSION}

A meticulous literature search suggests that this is the first dedicated study investigating the feasibility of synthetic MR imaging and brain volumetry in infants born very preterm, scanned at term- 

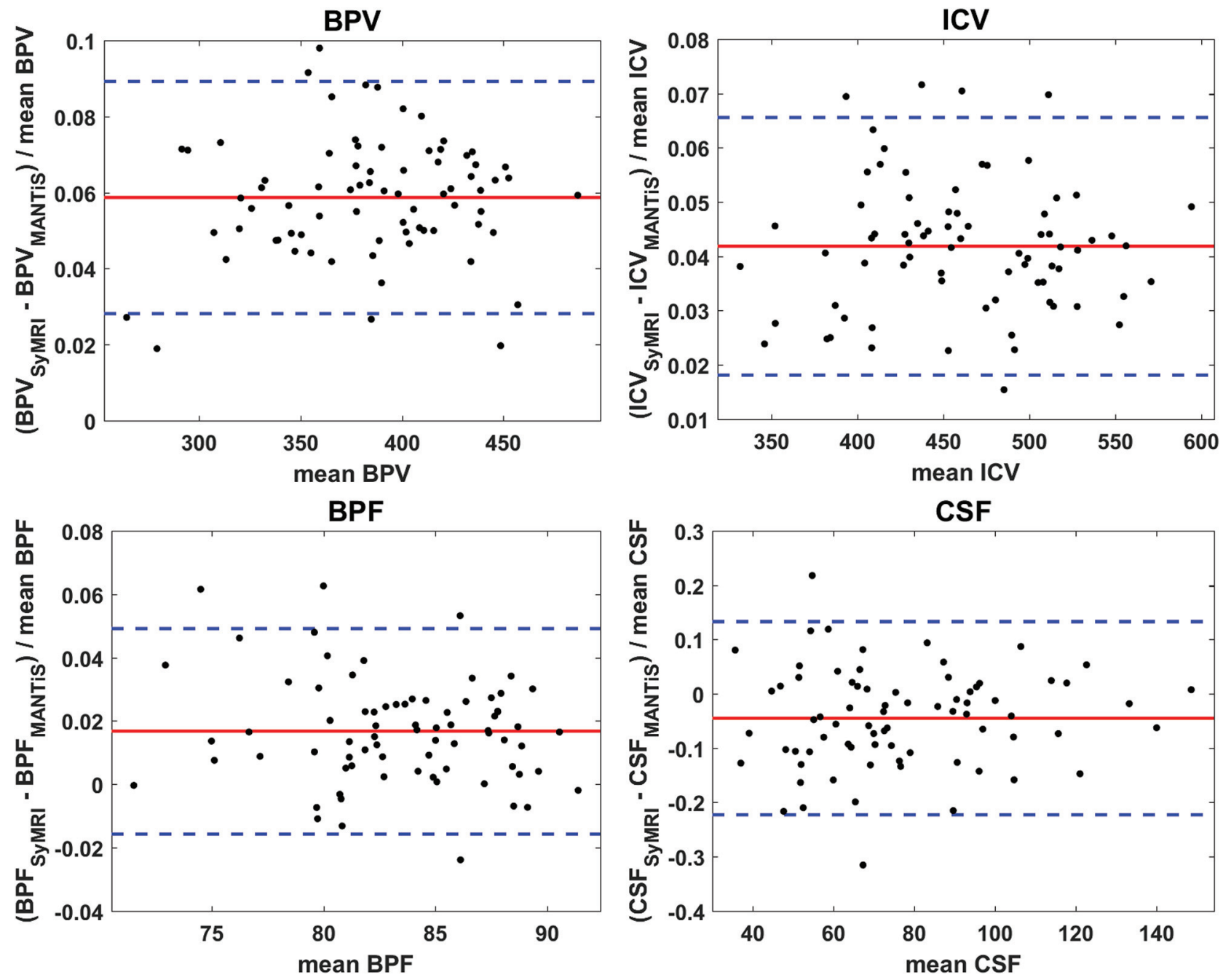

FIG 3. Bland-Altman plots comparing different volumetric metrics calculated with Synthetic MR imaging (labeled SyMRI) and MANTiS. The red line indicates the average relative difference, and the blue dashed lines indicate the average \pm 1.96 .

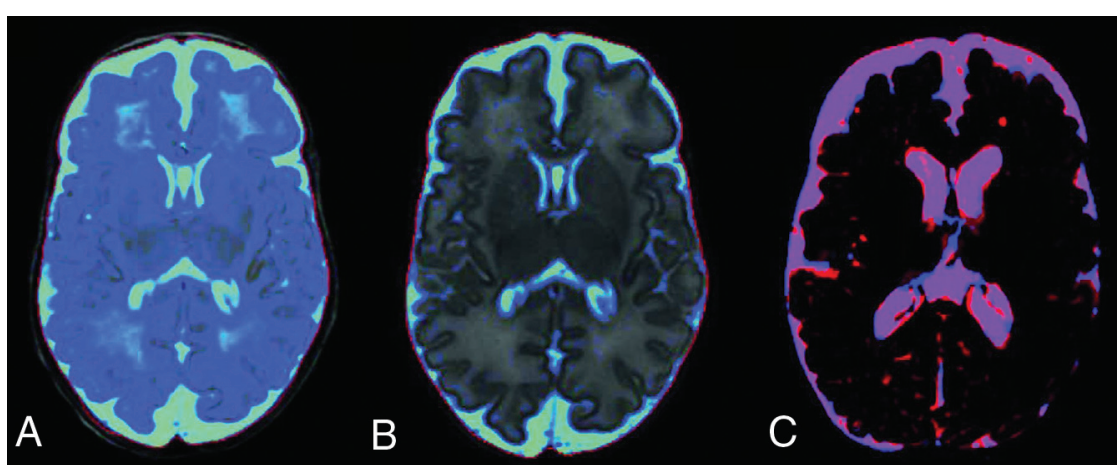

FIG 4. Synthetic CSF segmentation maps: SyMRI algorithm without optimization for the immature neonatal brain and CSF haze in the WM (blue) (A); improved SyMRI (Version 11.1) at the same level (B). Example of CSF overlap to calculate the Dice similarity coefficients (C). The synthetic CSF segment is red, and the MANTiS segment is blue. Regions where the segments overlap are purple. Voxels of the SyMRI CSF segment containing $<20 \%$ CSF are not shown. sufficient group. Both synthetic and conventional methods facilitated excellent visualization of normal brain structures. The accuracy of synthetic MR imaging for the detection of WM lesions was lower, but the difference was not significant $(P=1.00)$. Synthetic MR imaging sequences could therefore complement or possibly replace conventional T1WI and T2WI in neonatal imaging. Additionally, because the acquisition time remains approximately the same, contrasts that are currently omitted due to lack of time, such as single or double inverse recovery or proton density sequences, can be reconstructed without a time burden.

The inverted GM and WM conequivalent age. The quality of the synthetic images did not differ significantly from that of conventional images when the images were dichotomized into a good or excellent group and a poor or trast and the high amount of water in the neonatal brain render MR volumetrics in neonates challenging. ${ }^{11}$ Previous versions of SyMRI often mislabeled WM as CSF in neonates, resulting in 
inaccurate volumetric results. ${ }^{17,18}$ In the present study, a new version of SyMRI (Version 11.1) that features an optimized algorithm for neonatal brain segmentation was used. ${ }^{24}$ The volumetric measurements of synthetic MR imaging with this optimized algorithm were generally concordant with those of MANTiS software analysis. The Dice similarity coefficients exhibited excellent overlap for intracranial volume and BPV and moderate overlap for CSF. The Bland-Altman tables also indicated minimal spread between the 2 methods for intracranial volume, BPV, and brain parenchymal fraction. The segmentation of SyMRI was more robust than that of MANTiS, with respective failure rates of $6.6 \%$ and $11.0 \%$.

The added prognostic value of neonatal MR imaging volumetry has been demonstrated in numerous studies. ${ }^{6-8}$ To the best of our knowledge, SyMRI is the first commercially available product capable of accurately measuring brain volume in neonates. The tool is integrated into the radiology PACS system, and volume measurements are available in $<1$ minute.

The current study had several strengths, such as the evaluation of both the image quality and the volumetric segmentation results of synthetic MR imaging. The multidelay, multiecho sequence was part of the standard MR imaging protocol for all neonates born very preterm, which makes it an excellent representation for this population. All MR images were obtained very close to the termequivalent age ( $40 \pm 1$ week), which is considered optimal for the assessment of structural abnormalities that are related to long-term outcome. $^{25}$

The study also had some limitations. Because the children were scanned only once, it was not possible to evaluate the intramethod repeatability of volume segmentations. Second, although all the scans were carefully blinded for imaging method, the trained neuroradiologists may have been able to discern the difference between synthetic and conventional images with the naked eye, and this may have introduced some bias. Third, even though MANTiS is state-of-the-art research software for neonatal brain segmentation, it cannot be regarded as a criterion standard like manual segmentation. In the present study, the correspondence between the 2 methods was excellent. The improved SyMRI segmentation algorithm performed well when segmenting the brain parenchyma and delineating the brain mask, but in contrast to MANTiS, it is still unable to differentiate between GM and WM in neonates.

\section{CONCLUSIONS}

The current study suggests that in neonates, synthetic MR imaging can complement or be an alternative to conventional T1WI and T2WI sequences because it had excellent image quality and diagnostic performance. Volumetric measurements can be obtained in $<1$ minute after processing and are comparable with measurements derived via current state-of-the-art research methods. Synthetic MR imaging may therefore facilitate the routine use of these biomarkers in daily clinical practice, bridging the existing gap between the past decade of research on volumetric measurements and long-term outcomes and clinical practice.

Disclosures: Tim Vanderhasselt_UNRELATED: Employment: Radiologist, Comments: Staff Member Radiology Department Universitair Ziekenhuis Brussels, Brussels, Belgium. Maarten Naeyaert-UNRELATED: Employment: paid PhD student at the Bio-Imaging lab, Universiteit Antwerpen, through the Interdisciplinary PhD grant BOF UA 2012. Nina Watté—UNRELATED: Employment: radiology resident. Jeroen
Dudink - UNRELATED: Grants/Grants Pending: European Union-Innovative Training Network grant, Comments: project called Infans. https://www.tu-ilmenau.de/ infans/.* Hubert Raeymaekers-UNRELATED: Employment: Universitair Ziekenhuis Brussels. *Money paid to the institution.

\section{REFERENCES}

1. Pascal A, Govaert P, Oostra A, et al. Neurodevelopmental outcome in very preterm and very-low-birthweight infants born over the past decade: a meta-analytic review. Dev Med Child Neurol 2018;60:34255 CrossRef Medline

2. Spittle A, Orton J, Anderson PJ, et al. Early developmental intervention programmes provided post hospital discharge to prevent motor and cognitive impairment in preterm infants. Cochrane Database Syst Rev 2015;24:CD005495 CrossRef Medline

3. Woodward LJ, Anderson PJ, Austin NC, et al. Neonatal MRI to predict neurodevelopmental outcomes in preterm infants. $N \mathrm{Engl} \mathrm{J}$ Med 2006;355:685-94 CrossRef Medline

4. Alexander B, Murray AL, Loh WY, et al. A new neonatal cortical and subcortical brain atlas: the Melbourne Children's Regional Infant Brain (M-CRIB) atlas. Neuroimage 2017;147:841-51 CrossRef Medline

5. Balakrishnan U, Amboiram P, Ninan B, et al. MRI at term equivalent age for predicting long-term neurodevelopmental outcome in preterm infants: a cohort study. J Matern Fetal Neonatal Med 2020;33:1867-73 CrossRef Medline

6. Kidokoro H, Anderson PJ, Doyle LW, et al. Brain injury and altered brain growth in preterm infants: predictors and prognosis. Pediatrics 2014;134:e444-53 CrossRef Medline

7. Keunen K, Išgum I, van Kooij BJ, et al. Brain volumes at term-equivalent age in preterm infants: imaging biomarkers for neurodevelopmental outcome through early school age. J Pediatr 2016;172:88-95 CrossRef Medline

8. Gui L, Loukas S, Lazeyras F, et al. Longitudinal study of neonatal brain tissue volumes in preterm infants and their ability to predict neurodevelopmental outcome. Neuroimage 2019;185:728-41 CrossRef Medline

9. Kelly CJ, Hughes EJ, Rutherford MA, et al. Advances in neonatal MRI of the brain: from research to practice. Arch Dis Child Educ Pract Ed 2019;104:106-10 CrossRef Medline

10. Beare RJ, Chen J, Kelly CE, et al. Neonatal brain tissue classification with morphological adaptation and unified segmentation. Front Neuroinform 2016;10:12 CrossRef Medline

11. Išgum I, Benders MJ, Avants B, et al. Evaluation of automatic neonatal brain segmentation algorithms: the NeoBrainS12 challenge. Med Image Anal 2015;20:135-51 CrossRef Medline

12. Warntjes JB, Leinhard OD, West J, et al. Rapid magnetic resonance quantification on the brain: optimization for clinical usage. Magn Reson Med 2008;60:320-29 CrossRef Medline

13. West J, Warntjes JB, Lundberg P. Novel whole brain segmentation and volume estimation using quantitative MRI. Eur Radiol 2012;22:998-1007 CrossRef

14. Granberg T, Uppman M, Hashim F, et al. Clinical feasibility of synthetic MRI in multiple sclerosis: a diagnostic and volumetric validation study. AJNR Am J Neuroradiol 2016;37:1023-29 CrossRef Medline

15. Tanenbaum LN, Tsiouris AJ, Johnson AN, et al. Synthetic MRI for clinical neuroimaging: results of the magnetic resonance image compilation (MAGiC) prospective, multicenter, multireader trial. AJNR Am J Neuroradiol 2017;38:1103-10 CrossRef Medline

16. West J, Blystad I, Engström M, et al. Application of quantitative MRI for brain tissue segmentation at 1.5 $\mathrm{T}$ and 3.0 $\mathrm{T}$ field strengths. PLoS One 2013;8:e74795 CrossRef Medline

17. Kim HG, Moon WJ, Han J, et al. Quantification of myelin in children using multiparametric quantitative MRI: a pilot study. Neuroradiology 2017;59:1043-51 CrossRef Medline

18. McAllister A, Leach J, West H, et al. Quantitative synthetic MRI in children: normative intracranial tissue segmentation values during development. AJNR Am J Neuroradiol 2017;38:2364-72 CrossRef Medline

19. Andica C, Hagiwara A, Nakazawa M, et al. The advantage of synthetic MRI for the visualization of early white matter change in 
an infant with Sturge-Weber syndrome. Magn Reson Med Sci 2016;15:347-48 CrossRef Medline

20. Betts AM, Leach JL, Jones BV, et al. Brain imaging with synthetic MR in children: clinical quality assessment. Neuroradiology 2016;58:1017-26 CrossRef Medline

21. West $\mathrm{H}$, Leach JL, Jones BV, et al. Clinical validation of synthetic brain MRI in children: initial experience. Neuroradiology 2017;59:4350 CrossRef Medline

22. Lee SM, Choi YH, Cheon JE, et al. Image quality at synthetic brain magnetic resonance imaging in children. Pediatr Radiol 2017;47:163847 CrossRef Medline
23. Dice LR. Measures of the amount of ecologic association between species. Ecology 1945;26:297-302 CrossRef

24. Naeyaert M, Vanderhasselt T, Warntjes M, et al. Automatic brain segmentation in a neonatal population using a multi-delay multiecho sequence. In: Proceedings of the Annual Meeting and Exhibition of the International Society for Magnetic Resonance in Medicine, Paris France; June 16-21, 2018

25. Plaisier $\mathrm{A}$, Govaert $\mathrm{P}$, Lequin $\mathrm{MH}$, et al. Optimal timing of cerebral MRI in preterm infants to predict long-term neurodevelopmental outcome: a systematic review. AJNR Am J Neuroradiol 2014;35:84147 CrossRef Medline 